Geschmack $\rightarrow$ Giudizio, Geschmack, Geschmacksurteil

\title{
Giudizio, Geschmack, Geschmacksurteil
}

Sowohl das ital. giudizio im Sinne des Urteilsvermögens, als auch der Geschmack [G.] und das Geschmacksurteil [Gu.] dürften zu den wichtigsten, aber auch schwierigsten Begriffen jeder ästhetischen bzw. kunsttheoretischen Auseinandersetzung gehören. Alle drei Termini betreffen jenen komplexen Bereich ästhetischer Reflexion, in dem sich die Unbegrifflichkeit der Wahrnehmung und ihre begriffliche Formulierung berühren. Dabei geht die Forschung in der Regel von der etwas vereinfachenden Annahme aus, dass die Verwendung giudizio (bzw. frz. jugement, engl. judgement) seit dem 17. Jh. von gusto (frz. 
goût, engl. taste, dt. Geschmack) abgelöst worden sei (Klein 1970). Beide Begriffe zielen auf die Benennung eines Vermögens zur Beurteilung der Schönheit in den Künsten bzw. auf die Ausübung dieses Vermögens. Giudizio und gusto sind dabei nicht als Synonyme zu verstehen, sondern markieren gerade in ihrer Unterschiedlichkeit einen entscheidenden Wandel in der Einschätzung des Kunsturteils. Während das giudizio eher mit der Rationalität des Urteils und einem an Maßverhältnissen und Proportionen orientierten Kunstverständnis verbunden werden muss, ist der G. deutlicher auf die körperlich-sinnlichen Funktionen des Urteilenden, seine Subjektivität und seine Lust am Kunstwerk bezogen. Gerade der subjektive Anteil bleibt trotz vielfältiger Versuche einer Normierung des G. konstituierend für die unterschiedlichen historischen Konzeptionen des $\mathrm{Gu}$. Mit der Durchsetzung des G.-Begriffs vollzieht sich daher auch der Übergang von der kunsttheoretischen zur ästhetischen Reflexion über Kunst.

\section{Antike}

Der lateinische Begriff iudicium, auf den das italienische giudizio zurückgeht, ist zunächst ein juristischer Terminus, der sich aus der Deutung des Rechts, ius dicere, herleitet. Da er sich auf die richtige Einschätzung einer Situation oder eines Sachverhaltes bezieht, verbindet sich mit dem Urteil zugleich ein Wahrheitsanspruch. Im Bereich der antiken Rhetorik, die nicht allein auf die Rede vor Gericht beschränkt ist, meint das iudicium neben der richtigen Beurteilung des jeweiligen Falls auch die Beurteilung der Rede selbst. Er bezeichnet daher sowohl die Fähigkeit des Urteilens im Sinne eines bestimmten Vermögens, d.h. die Urteilskraft, als auch die Ausübung dieser Fähigkeit und ihr Resultat, das als ein Werk des richtigen Urteilens begriffen wird. Dabei ist der Redner, wie Cicero (106-43 v.Chr.) im Orator ausführt, immer schon dem iudicium seines Publikums ausgesetzt: "So oft wir nämlich reden, so oft wird ein Urteil über uns gefällt. « Neben dem Urteil in der Sache kann sich dieses auch auf den Stil der Rede beziehen, wobei sich mit letzterem Genuss und Vergnügen an den sprachlichen und formalen Qualitäten verbinden. Das Urteil gründet sich in allen Fällen auf den sensus, d.h. die sinnliche Wahrnehmung, wird aber erst durch eine gleichzeitige Operation des Verstands möglich, wobei der gute Redner dem je unterschiedlichen Verhältnis von Sinnlichkeit und Vernunft stets Rechnung trägt. Das richtige Urteil ist, weil es sich wesentlich auf Erfahrung gründet, zwar sowohl in seinen sachlichen als auch formalen
Aspekten einübbar, ohne eine angeborene Begabung aber nicht zu vermitteln.

Welche unterschiedliche Relevanz dem Urteil des Einzelnen beigemessen werden kann und welche sozialen Differenzen dabei zum Tragen kommen, zeigt sich an einer von Plinius (61-112 n.Chr.) kolportierten Anekdote über den antiken Maler Apelles, bei der das Problem des richtigen Urteils und seiner Angemessenheit explizit auf ein Werk der bildenden Kunst angewandt wird. Apelles soll seine Gemälde bewusst dem Urteil der Offentlichkeit ausgesetzt haben, wobei er aufgrund der Einwände eines Schusters zwar die Sandale der von ihm dargestellten weiblichen Gestalt änderte, als der Kritiker aber am nächsten Tag wiederkehrte, um jetzt die Schönheit der Figur selbst anzuzweifeln, soll er dessen Urteilsfähigkeit in Fragen der Schönheit mit den sprichwörtlichen Worten: "Schuster, bleib bei Deinem Leisten" zurückgewiesen haben (Naturalis historia, 35, $84 \mathrm{f}$.). Mit der hier deutlich werdenden Frage nach dem Subjekt des Urteils und seiner sozialen Durchsetzungskraft eröffnet sich im Bereich der Künste nicht nur das historisch bis in die Moderne umkämpfte Problem einer Unterscheidung zwischen Laie und Kenner. Sichtbar wird auch die von P. Bourdieu vor allem im Hinblick auf das 20. Jh. betonte Funktion der Kunst als Feld sozialer Distinktion.

\section{Frühe Neuzeit}

Anders als in der Antike richtet sich das Interesse der mittelalterlichen philosophischen Tradition in erster Linie auf das logische und nicht auf das ästhetische Urteil. Der Begriff des iudicium bzw. giudizio kann erst im Zusammenhang mit der Rezeption der antiken Rhetorik, wie sie sich seit dem 15. Jh. durchgesetzt hat, in den Bereich der bildenden Kunst und der Kunsttheorie vordringen. Das Urteil bzw. die Urteilskraft ist dabei wie in der Rhetorik eine Kategorie, die sowohl dem Künstler als auch dem über das Kunstwerk urteilenden Publikum zukommen muss. Entsprechend empfiehlt L.B. Alberti (1404-1472) in seinem Traktat De Pictura, die eigenen Werke bewusst dem kritischen Urteil der Freunde auszusetzen. Auch für Leonardo da Vinci (1452-1519) determiniert das giudizio den Werkprozess, denn nur wenn das Urteil des Künstlers das ausgeführte Werk übertrifft, wird dieser sein Werk immer weiter verbessern: "quando il giuditio supera l'opera, essa opera mai finisce di migliorare." Übertrifft dagegen das Werk das Urteil, wird dies von Leonardo gegen den Künstler und sein Werk geltend gemacht, womit der vom Künstler vollzogene Akt des Urteilens als die maß- 
gebliche Instanz klassifiziert und gegenüber dem Werk privilegiert wird. In Anlehnung an die Physiologie des Aristoteles versteht Leonardo das giudizio als eine Operation des Gemeinsinns, d.h. des sog. sensus communis. Dieser Gemeinsinn gilt als ein im Gehirn lokalisierbares Vermögen, in dem alle Sinnesdaten zusammengetragen werden. Sein Sitz im Gehirn, den Leonardo in einigen Zeichnungen anatomisch zu bestimmen versucht, bedingt eine physiologische Nähe zu den Augen, so dass dem Sehen ein privilegierter Zugang zum sensus communis und damit auch zum richtigen Urteil zugeschrieben werden kann: "Das Auge empfängt die Bilder [species] [...] der Gegenstände, von dort gelangen sie zur impressiva, und von dort zum sensus communis, und dort werden sie beurteilt" (vgl. Summers 1987).

Dem mit dem erkenntnistheoretischen Primat des Sehens verknüpften Urteil des Auges, giudizio dell' occhio, sind sowohl der Künstler als auch die verständigen Kunstbetrachter verpflichtet. Ob die Fähigkeit des richtigen Urteils grundsätzlich als erlernbar und d.h. als rational und an einer Schönheit der Proportion orientiert - oder als angeboren gilt, bleibt im Rahmen der Kunst- und Architekturtheorie der Frühen Neuzeit umstritten, wobei auch Architekturtheoretiker wie S. Serlio in dieser Frage eine vermittelnde Position angenommen haben. Dass dem Künstler ein besonderes, die Maßverhältnisse gerade verletzendes Urteilsvermögen der Augen zukommen kann, spiegelt sich in einer durch G. Vasari (1511-1574) überlieferten Auseinandersetzung um die Verbindlichkeit der Einhaltung der mathematisch exakten Proportionen. Danach soll Michelangelo (1475-1564) die dem Schein dienenden Verkürzungen eines Reliefs und die daraus resultierende Vernachlässigung der Maße mit den Worten begründet haben wdass man den Zirkel in den Augen haben müsse und nicht in der Hand, [denn allein] das bedeute, giudizio zu haben «. Wie unterschiedlich der Terminus giudizio gefüllt werden konnte und gerade durch seine Unbestimmtheit als ein Platzhalter in den künstlerischen Auseinandersetzungen diente, zeigt sich in den Angriffen, die Annibale Carracci (1560-1609) gegen G. Vasari erhoben hat, den er als "voller Affektiertheit und ohne jedes Urteilsvermögen « beschimpft, obwohl dieser selbst dem richtigen Urteil einen hohen Stellenwert einräumte.

Noch im 17. Jh. bleibt das jugement eine entscheidende Kategorie in der Beurteilung von Kunstwerken, wie sich etwa an den Äußerungen N. Poussins (1594-1665) aufzeigen lässt. Schon seit ca. 1580 kommt es in Italien und Spanien bei der Bezeichnung des entsprechenden Vermögens aber zu einer Ergänzung und zum Teil auch Ablösung des giudizio durch die subjektivere Rede vom gusto. Das erkenntnistheoretische Primat des Sehens wird damit in Bezug auf die Schönheit und für den Bereich der bildenden Kunst, Literatur und Musik durch die Orientierung am Geschmackssinn aufgegeben. G.P. Lomazzo (1538-1600), für den giudizio auf dem Unterscheidungsvermögen, der discrezione basiert, verwendet beispielsweise beide Begriffe in syntaktischer Reihung, wenn er vom "giudizio o gusto « des Künstlers spricht. Der gusto scheint hier eher der persönlichen Neigung zu entsprechen. Sowohl in Italien wie auch Spanien wird der gusto von einer Reihe Autoren dann vor allem für den Bereich moralphilosophischer Fragen verwendet, wie sie aus der neuen höfischen Kultur resultierten, wobei hier auf die christliche Ausdeutung des sapor als einer Form intuitiver Erkenntnis zurückgegriffen wird. Gerade in Spanien wird dabei auch die semiotische Nähe zwischen justo und gusto betont.

\section{Aufklärung}

1712 schrieb J. Addison (1672-1719) in der Zeitschrift Spectator: »Da der Begriff [taste] im Gespräch sehr häufig vorkommt, möchte ich seinen Sinn klarstellen." Die Begriffsklärung erscheint ihm notwendig, um die "Vergnügen der Einbildungskraft", denen er in den folgenden Monaten mehr als zehn Essays widmen wird, einzuleiten. Geschmack definiert er als "jene Fähigkeit der Seele, die in der Lage ist, die Schönheiten eines Autors mit Vergnügen, seine Schwächen mit Mißfallen zu erkennen.« Als Erfinder der metaphorischen Verwendung des G.Begriffs bezeichnet Addison den spanischen Moralphilosophen B. Gracian (1601-1658), dessen Bücher seit dem späten 17. Jh. ins Englische und Französische übertragen wurden. Die verbreitete Auffassung, dass hier der Ursprung der Metapher vom `G. als einem ethischen und ästhetischen Vermögen liege, konnte erst in der jüngeren Forschungen korrigiert werden, ohne dass dadurch die Bedeutung, die den Schriften Gracians in der Übermittlung des neuen Terminus zukommt, in Frage gestellt wäre (vgl. Frakkowiak 1994; Russo 2000).

Addison stellt eine Verbindung her zwischen dem literarischen taste und dem ebenfalls lustvollen Sinnesvermögen des physiologischen G.-Sinnes. Diese Annahme einer Vergleichbarkeit der ästhetischen Kategorie des G. mit der Zunge als dem Organ der G.Wahrnehmung werden die meisten Autoren des 18. Jh., die sich in einer fast unübersichtlichen Fülle von Schriften zur Problematik des G., seiner Ausbildung und seiner Verderbnis widmen, mehr oder 
weniger explizit teilen. $\mathrm{Zu}$ den wichtigsten Autoren der die europäischen Literaturen bestimmenden Debatte gehören unter anderem Ch. Batteux (1713-1780), F. Hutcheson (1694-1746), A. Gerard (1728-1795), Voltaire (1694-1778), Montesquieu (1689-1755), J.B. d'Alembert (1717-1783), A. Baumgarten (1714-1762), D. Hume (1711-1776).

Die Herausbildung und Kultivierung des G. ist im 18. Jh. Teil einer sozialen Praxis, die eng mit der Entstehung historisch neuer sozialer Schichten verbunden ist. Der Zusammenhang zwischen den Konzeptionen des G. und bestimmten sozialen Verhaltensformen deutet sich bereits im gran goût des französischen Klassizismus an. Dass Sozialität und guter G. als eng verbunden gedacht werden, betont etwa auch Voltaire in seinen Äußerungen zum goût in der Encyclopédie; "Quand il y a peut de societé [...], il n'a pas deqoui se former le goût. « Die wesentliche Rolle des G. in der Kultur des 18. Jh. ließe sich auch anhand der Einleitung der Encyclopédie darlegen, wo dem G. und seiner Funktion breiter Raum gewährt wird, so dass er in den Kontext der erkenntnistheoretischen, gesellschaftlichen und ästhetischen Überzeugungen des gesamten philosophischen Unternehmens gestellt werden muss. Gerade vor diesem Hintergrund einer Relation zu gesellschaftlichen Fragen wird es verständlich, warum der G. im 18. Jh. zu einem der zentralsten Termini der ästhetischen Diskussion werden konnte. Aufgrund der Einschätzung des G. als ein Ergebnis zivilisatorischer Leistung und seiner daraus resultierenden Verbindung mit bestimmten Gesellschaftsformen richtet sich das Interesse auch auf die Möglichkeit seiner Bildung und Formung. Montesquieu, Theoretiker der Freiheit und des Gesetzes, hat in seinem 1757 postum erschienen Essay sur le goût zwischen natürlichem und erworbenem G. unterschieden, wobei er die Spontaneität des goût naturel gegenüber der Erlernbarkeit des goût acquis betont. Wie Voltaire im gleichen Artikel ausführt, reicht es aber ihm Hinblick auf die Erlernbarkeit gerade nicht aus, die Schönheit eines Werkes zu kennen oder zu sehen, vielmehr muss man durch sie berührt werden - wil faut la [la beauté] sentire, en être touché«. Mit dem G. verknüpft sich daher die sensibilité des wahrnehmenden Subjektes.

Gerade weil der G. zunehmend als ein passives Vermögen klassifiziert wird, das sich von der Aktivität des Genies unterscheidet, kann die ästhetische Diskussion um den G. bis in die Debatten des 18. Jh. über das Verhältnis und die Rollen der Geschlechter vordringen (Jones 1998). Das weibliche Geschlecht und seine Schönheit sollen mit der Kultivierung des Geschmacks verknüpft werden, wobei weiblich konnotierte Schönheit und männlich konzipiertes Genie bzw. männliche Erhabenheit gegeneinander ausgespielt werden. Frances Reynolds, Schwester des Malers Sir J. Reynolds, schreibt in ihrem Traktat An Enquiry Concerning the Principles of Taste, der 1785 publiziert wurde: „Die Pflege der gesellschaftlichen und moralischen Empfindungen ist die Pflege des G. und die häusliche Sphäre ist der wahre und fast einzige Bereich, in dem der G. in seiner höchsten Würde zur Erscheinung kommen kann und seinen wahren Glanz zeigt. Dies ist besonders dem weiblichen G. angemessen.*

Fast parallel mit der Rede vom G. und seiner Fähigkeit zur Wahrnehmung des Schönen verstärkt sich die häufiger weniger explizite, aber dennoch intensive Beschäftigung mit dem Abstoßenden und Degoutanten. Eine elaborierte Debatte um den Ekel prägt unterschwellig die Jahre seit 1750 , in deren Verlauf sich u. a. J.E. Schlegel (1719-1749), Mendelssohn (1729-1786), Lessing (1729-1781), Herder (1744-1803) und Kant (1724-1804) zu Wort melden (Menninghaus 1999). Ekel und Barbarei können daher im 18. Jh. als die wichtigsten Gegenbegriffe zum G. gelten, wobei vom Ekel gerade wegen der Stärke seiner Empfindung eine besondere, sich bis in die Ästhetiken der Moderne fortsetzende Faszination auszugehen scheint.

Welche Aporien sich aus der Abgrenzung des guten G. vom barbarisch schlechten ergeben, hat D. Hume in seinem kurzen Essay On the Standart of Taste von 1757 in sprachlicher und argumentativer Brillanz dargelegt: "Wir sind geneigt, alles das als barbarisch zu bezeichnen, was sich von unserem eigenen $\mathrm{G}$. und Verständnis stark unterscheidet: Bald aber finden wir die Formulierung dieses Vorwurfs gegen uns selbst gerichtet. Z Zum Problem wird für den skeptischen Empiristen Hume nicht nur die Verbindlichkeit des Gu., sondern auch die Frage nach seiner Diskursivierbarkeit. Die ursprünglich physiologisch gemeinte paradoxe Behauptung, dass sich über den G. (nicht) streiten ließe, wird damit in neuer Form aufgenommen und am Ende zugunsten einer universellen, begrifflich vermittelbaren Geltung entschieden. Gerade auf Grund der Einsicht in die Subjektivität des ästhetischen Urteils beharrt Hume auf dem verbindlichen Standard eines historischen und historische Grenzen überschreitenden G., um so einerseits die drohende Spannung zwischen Subjektivität und Gesellschaft aufzuheben und andererseits ganz grundsätzlich an der philosophischen Relevanz von Erfahrung festhalten zu können.

Mit I. Kants 1790 erschienener Kritik der Urteilskraft, durch die er sein Projekt einer kritischen Philosophie zum Abschluss bringen konnte, kommt es am Ausgang des Jahrhunderts zu einer für die Geschichte 
der Ästhetik folgenreichen Neubestimmung des Gu. Kant muss einräumen, dass gerade das ästhetische Urteil, das Schönheit und Erhabenheit wie Natur oder Kunst betreffen kann, im Hinblick auf die Frage nach den ihm zugrundeliegenden Prinzipien als problematisch anzusehen sei. Dabei bestimmt er Lust und Unlust als "das Rätselhafte der Urteilskraft, wobei er betont: „Ohne Beziehung auf das Gefühl des Subjektes ist die Schönheit eines Gegenstandes nichts." Indem er einen bloßen "Sinnengeschmack " von "Reflexionsgeschmack « unterscheidet, kann er das bloß Angenehme, das mit einem bestimmten Interesse verbunden ist, vom Schönen absetzen. Als einen der "Schlüssel" zur Kritik des G. gilt ihm die Frage, ob das Gefühl der Lust der Beurteilung des Gegenstandes vorausgeht oder ihr folgt (Kritik der Urteilskraft, \$9): „Die subjektive allgemeine Mitteilbarkeit der Vorstellungsart in einem Geschmacksurteil $[\ldots]$ kann nicht anders als der Gemütszustand in dem freien Spiel der Einbildungskraft und des Verstandes sein, indem wir uns bewusst sind, dieses zum Erkenntisse überhaupt schickliche subjektive Verhältnis ebensowohl für jedermann gelten und folglich allgemein mitteilbar sein müsse, als es eine jede bestimmte Erkenntnis ist, die doch immer auf jenem Verhältnis als subjektiver Bedingung beruht. Diese bloß subjektive (ästhetische) Beurteilung des Gegenstandes, oder der Vorstellung, wodurch er gegeben wird, geht nun vor der Lust an dem selben vorher, und ist der Grund dieser Lust an der Harmonie der Erkenntnisvermögen." Die Lust, die dem Urteil über das Schöne zugrunde liegt, ist demnach eine Lust am Spiel unseres Erkenntnisvermögens, bei dem wir uns reflektierend auf die Form unseres Erkennens beziehen (Kern 2000).

Mit der stark am Begriff der Reflexion ausgerichteten frühromantischen Kunstkritik verliert der Terminus G. um 1800 wesentliche Aspekte seiner Relevanz, wenn er auch weiter zum begrifflichen Arsenal der ästhetischen Debatten einer bürgerlichen Kultur gehört. Erst in jüngerer Zeit scheint das philosophische, kunsthistorische und literaturwissenschaftliche Interesse wieder verstärkt auf jener Kategorie zu liegen, die sich mit der Metapher des G. verbindet, nämlich der Erfahrung eines spezifischen Lustempfindens, das nur mit dem Ästhetischen als Spiel möglich wird (Kern 2000).

$\rightarrow$ Ästhetik; Genie; Grazia; Klassik und Klassizismus; Wahrnehmung

\section{Literatur}

R. KLeIn, Giudizio e gusto dans la Théorie de l'art au Cinquecento. In: Ders., La forme e l'intelligible. Ecrits sur la Renaisance et l'art Moderne, Paris 1970, 341-352. -
G. Agamben, Gusto. In: Encyclopedia Einaudi, Bd. 6, Turin 1977, 1019-1038. - P. Bordieu, Die feinen Unterschiede. Kritik der gesellschaftlichen Urteilskraft, Frankfurt a.M. 1982 [zuerst frz. 1979]. - D. Summers, The Judgement of Sense. Renaissance Naturalism and the Rise of Aesthetics, Cambridge 1987. - H. CAYGILL, The Art of Judgement, Oxford 1989. - U. Frackowiak, Der gute G. Studien zur Geschichte des Geschmacksbegriffes, München 1994. - R. W. JonES, Gender and the Formation of Taste in Eighteenth-Century Britain. The analysis of beauty, Cambridge 1998. - W. MENNINGHaUs, Ekel. Theorie und Geschichte einer starken Empfindung, Frankfurt a.M. 1999. - R. LÜthe/ M. Fontius, G./ Gu. In: K. BArcK u.a. (Hg.), Ästhetische Grundbegriffe, Bd. 2, Stuttgart 2001, 792-819. - L. Russo (HG.), Il Gusto. Storia di un idea estetica (Aesthetica; 52), Palermo 2000. A. KERN, Schöne Lust. Eine Theorie der ästhetischen Erfahrung nach Kant, Frankfurt a.M. 2000, - H. BAADER, G. Zur frühen Geschichte eines ambivalenten Konzeptes. In: Zeitschrift für Kunstgeschichte [im Druck].

Hannah Baader 